\title{
Situación actual de la rehabilitación cardiaca en Chile
}

\author{
CLAUDIO SANTIBÁÑEZ1 ${ }^{1}$, CARMEN PÉREZ-TERZIC ${ }^{2}$, \\ FRANCISCO LÓPEZ-JIMÉNEZ², MERY CORTÉS-BERGODERI ${ }^{2}$, \\ MARÍA VIRGINIA ARAYA ${ }^{1}$, GERARD BURDIAT ${ }^{3}$
}

\section{Current status of cardiac rehabilitation in Chile}

Background: Cardiac rehabilitation (CR) programs play an important role in the control and prevention of new cardiac events. Aim: A survey was performed to evaluate the current situation of CR programs in Chile. Material and Methods: A questionnaire evaluating the structure of rehabilitation centers, characteristics of the rehabilitation programs and patients, management of risk factors, reimbursement methods, human resources and potential barriers for an efficient rehabilitation, was mailed to centers dedicated to CR in Chile. Results: Eight centers were contacted and seven responded. Coronary heart disease is the most common underlying disease of attended patients and CR is carried out mainly during phases II and III. All CR centers perform an initial assessment, stratify patients, plan and provide tips on physical activity and nutrition. Only three centers provide help to quit smoking. Lipid profile and blood sugar are assessed in $62 \%$ of centers. Most practitioners involved are cardiologists, nurses, physiotherapists and nutritionists, all trained in cardiopulmonary resuscitation. The main barrier for their development is the lack of patient referral from practitioners. Conclusions: Despite the recognized value of $C R$ in the care of patients after a cardiac event, this study reveals the need for further development of such programs and improvement of patient referrals.

(Rev Med Chile 2012; 140: 561-568).

Key words: Coronary artery disease; Rehabilitation, cardiac; Secondary prevention.

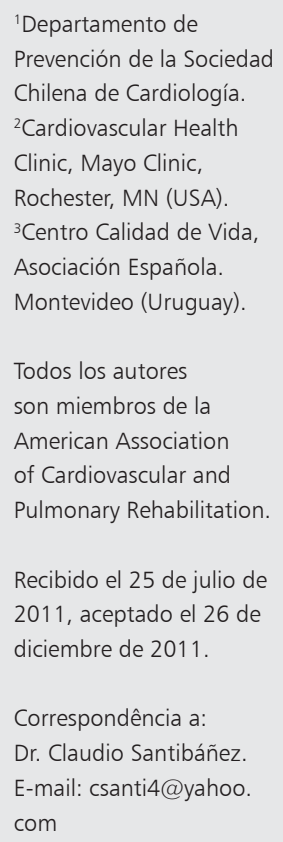

N os encontramos inmersos en una epidemia de enfermedades vinculadas a cambios de hábitos y estilos de vida. Estos serán los causantes de muchas de las patologías que la OMS ha calificado como "Enfermedades Crónicas No-Trasmisibles" (ECNT) ${ }^{1-4}$.

Chile no escapa a esta problemática y está demostrado que las enfermedades cardiovasculares son la primera causa de mortalidad. Ellas han crecido rápidamente como resultado del tabaquismo, el tipo de alimentación, la urbanización y el sedentarismo ${ }^{5-9}$.

Los cambios en el estilo de vida se dan en un contexto conocido hoy como "transición demográfica y de salud".

En Chile, la encuesta de vida saludable patrocinada por el Ministerio de Salud da muestra de la critica situación que se vive, con índices de sedentarismo de $88,6 \%$, exceso de peso $67 \%$, tabaquismo $40,6 \%$, dislipemia $38,5 \%$, hipertensión arterial $26,9 \%$, diabetes $9,4 \%{ }^{10,11}$.

Lo primordial es prevenir la aparición de las patologías cardiovasculares, sin embargo, una vez producido el evento coronario, su manejo, incluyendo mejoras en el estilo de vida, es una urgente medida a desarrollar. Dado el aumento de la sobrevida de estos pacientes, gracias a los avances en el tratamiento precoz, se hace imperioso implementar programas de prevención integrales para evitar las recaídas y mejorar el costo-eficacia de dichos procedimientos. En este aspecto, la rehabilitación cardiovascular (RC) es considerada pilar terapéutico fundamental ${ }^{12-14}$. 
La rehabilitación cardiaca (RC) es un término establecido por la OMS en 1964 y comprende un programa multidisciplinario y estructurado de ejercicios físicos, consejería especializada e individualizada designado para la recuperación del enfermo cardiaco. Está dividido en fases y tiende a la optimización del control de los FR, consejo nutricional, soporte psicológico y social, buscando estabilizar la enfermedad y evitar su progresión, mejorando la morbi-mortalidad ${ }^{15}$.

A pesar del impacto del tratamiento farmacológico, revascularización intervencionista o quirúrgica u agentes trombolíticos que han hecho disminuir la mortalidad en eventos coronarios agudos, lamentablemente la mortalidad posterior al primer infarto permanece similar a lo publicado en el estudio Framingham que es de 36\% a los 5 años en los hombres y de $51 \%$ en las mujeres ${ }^{9,14}$.

El beneficio de la RC en el pronóstico de pacientes con enfermedad cardiovascular se ha demostrado en varios estudios y es evidencia Clase I de todas las guías actuales sobre el manejo del paciente luego de un evento coronario y comprende el manejo completo de todos los factores de riesgo modificables $^{14}$.

En Chile se publicó en el año 2000 acerca de los beneficios de la $\mathrm{RC}^{16}$. El propósito de este trabajo es describir la situación actual de la RC en Chile, cómo se lleva a cabo, la implementación de sus programas, qué componentes tiene, cuál es el grupo de profesionales a cargo, y cuáles son las principales limitantes para que se desarrolle con éxito.

\section{Material y Métodos}

Se realizó un estudio descriptivo a través del análisis de datos recogidos provenientes de un cuestionario común para todos los países de Sudamérica, que, luego de ser aprobado para su utilización por el Comité Latinoamericano de Prevención y Rehabilitación Cardiovascular, fue distribuido en todos los países sudamericanos. La encuesta incluye preguntas de opción múltiples y abiertas para recopilar información cuantitativa y cualitativa. Los temas abordados por la encuesta incluyen 1) información general; 2) la estructura de cada centro de CRP; 3 ) las características de cada CRP (por ejemplo, el número de sesiones, intensidad del ejercicio, información sobre pro- veedores de servicios médicos, servicios ofrecidos y seguimiento de protocolos); 4) la población asistida; 5) estrategias para la administración de factores de riesgo de enfermedades cardiovasculares; 6) información sobre el reembolso; 7) recursos humanos; 8) potenciales barreras para el programa; 9) la existencia de una base de datos clínico o de investigación de pacientes; y 10) ofrecen otros servicios cardiovasculares en el centro. La validez de contenido se basa en los resultados de una encuesta preliminar, cuyos resultados han sido publicados con anterioridad. Expertos coinvestigadores de las encuestas (FLJ) inicialmente evaluaron la validez interna y fiabilidad de los elementos a través de amplias reseñas ${ }^{20}$. También utilizamos grupos focales que examinaron la intención de la encuesta general y para determinar si o no la información prevista podría ser recogida por los temas propuestos. Elementos individuales fueron revisados varias veces hasta que hubo consenso sobre su validez interna. Reproducibilidad no fue evaluado sistemáticamente dada la sencillez de las preguntas.

Cada cuestionario fue enviado a un responsable de cada Sociedad de Cardiología para ser distribuidos a los centros de RC locales vigentes y avalados por la Sociedad Chilena de Cardiología ${ }^{17}$.

En Chile se envió a todos los centros que realizan RC vía correo electrónico, para ser llenados y devueltos al responsable del estudio. De los centros donde no se obtuvo respuesta se les reenvió el correo o se les contactó telefónicamente para conocer los motivos de la no respuesta y su situación actual. Los cuestionarios completados fueron enviados para su procesamiento a la Clínica Mayo en Rochester, MN (Estados Unidos de Norteamérica), quien auspició como Institución de apoyo.

Se utiliza estadística descriptiva con frecuencias y porcentajes.

Para determinar la cantidad de pacientes candidatos a RC, se recurre a opinión de expertos y a la extrapolación de los datos del registro nacional de infarto (Registro GEMI) el cual cubre 25\% del total de los pacientes con algún accidente coronario agudo ${ }^{18}$.

\section{Resultados}

En Chile funcionan 8 centros que ofrecen el servicio de RC. De ellos, 7 llenaron y completaron la encuesta (Clínica Alemana Puerto Varas, Clínica 
Alemana de Santiago, Centro de Rehabilitación Cardiovascular Prof. Doctor Rafael del Río de la Torre de CRS Peñalolén Cordillera Oriente, Departamento Cardiovascular Hospital Clínico Universidad de Chile, Cardiología Preventiva y Rehabilitación Cardiovascular PUC, Complejo Hospitalario San José CDT Dra. Eloísa Díaz, Cardiokines Clinics).

Tres de los 7 centros encuestados son entidades públicas.

El número de pacientes promedio vistos al año por centro en Chile fue de 153, lo cual si se relaciona con el número total de pacientes que sufren un accidente coronario agudo en un año nos da una cifra cercana a $5 \%$ de pacientes que reciben rehabilitación.

La Fase I de la RC, la realiza $42 \%$ de los centros, la Fase II el 100\% la Fase III, 71\%. De los que realizan la Fase II, sólo $14 \%$ logra comenzar antes de las 2 semanas del alta y la mayoría cuenta con monitorización telemétrica. Ochenta y cinco porciento realiza 3 sesiones semanales con una duración promedio de 12 semanas (Tabla 1).

El personal que forma parte del equipo de trabajo fue similar en todos los centros.

Para calcular la intensidad del ejercicio la mayoría prefiere la utilización del consumo de oxígeno por medición indirecta en Mets obtenido de una prueba ergometría convencional. Esta se realizaba en $100 \%$ en Fase II y IV y $80 \%$ en Fases III.

Todos contaban con equipos multidisciplinarios de profesionales. El cardiólogo estaba presente en todos. Participaban también enfermera, kinesiólogo, nutricionista, trabajadora social. Las consultas a psicólogo se realizaba en 75\% de los centros. Todos los profesionales participantes recibieron entrenamiento en reanimación cardiopulmonar básica (Tabla 2).

La totalidad de los centros (100\%) estaba en condiciones y realizaba evaluación inicial del paciente, pesquisando los factores de riesgo y estratificando a los mismos. Todos planificaban, brindaban consejos y se entrenaba al personal y a los pacientes en la toma de la frecuencia car-

\section{Tabla 1. Del total de centros, de sus fases y} sus respectivos componentes

\begin{tabular}{|c|c|}
\hline $\begin{array}{l}\text { Fase I (porcentaje de centros que la realizan) } \\
\text { Que profesionales realizan la fase I } \\
\text { Fisioterapeuta o kinesiólogo }\end{array}$ & $100 \%$ \\
\hline Fase II (porcentaje de centros que la realizan) & $100 \%$ \\
\hline $\begin{array}{l}\text { Características de la fase II } \\
\text { Comienzo posterior a } 2 \text { semanas del egreso } \\
\text { Monitorización ECG }\end{array}$ & $\begin{array}{l}71 \% \\
71 \%\end{array}$ \\
\hline $\begin{array}{l}\text { Número de sesiones semanales promedio } \\
\text { * } 1 \text { sesión } \\
\text { * } 2 \text { sesiones } \\
\text { * } 3 \text { sesiones } \\
\text { * }>3 \text { sesiones }\end{array}$ & $\begin{array}{l}0 \\
14 \% \\
85 \% \\
14 \%\end{array}$ \\
\hline $\begin{array}{l}\text { Duración de la fase II en semanas (media) } \\
\text { Recursos o maquinas }\end{array}$ & 12 semanas \\
\hline $\begin{array}{l}\text { * Banda sin fin } \\
\text { * Bicicleta estática } \\
\text { * Pesas ligeras } \\
\text { * Banco de gimnasia } \\
\text { * Equipos multifuerza } \\
\text { * Marcha, caminata }\end{array}$ & $\begin{array}{r}100 \% \\
100 \% \\
100 \% \\
56 \% \\
71 \% \\
100 \%\end{array}$ \\
\hline $\begin{array}{l}\text { Que herramienta utilizan para el cálculo de la } \\
\text { intensidad del ejercicio }\end{array}$ & \\
\hline $\begin{array}{l}\text { * Borg } \\
\text { * Mets } \\
\text { * Edad } \\
\text { * VO } \\
\text { * Frecuencia cardiaca }\end{array}$ & $\begin{array}{r}71 \% \\
100 \% \\
85 \% \\
28 \% \\
14 \%\end{array}$ \\
\hline $\begin{array}{l}\text { Que profesionales realizan la fase II } \\
\text { * Médico } \\
\text { * Enfermera } \\
\text { * Fisioterapeuta o kinesiólogo }\end{array}$ & $\begin{array}{r}85 \% \\
56 \% \\
100 \%\end{array}$ \\
\hline $\begin{array}{l}\text { Fase III (porcentaje de centros que la realizan) } \\
\text { Que herramienta utilizan para el cálculo de la } \\
\text { intensidad del ejercicio } \\
\text { * Borg } \\
\text { * Mets } \\
\text { * Edad } \\
\text { * } \mathrm{VO}_{2} \\
\text { * FC }\end{array}$ & $\begin{array}{l}40 \% \\
80 \% \\
60 \% \\
20 \% \\
12,5 \%\end{array}$ \\
\hline $\begin{array}{l}\text { Que profesionales realizan la fase III } \\
\text { * Médico } \\
\quad \text { * Enfermera } \\
\text { * Fisioterapeuta o kinesiólogo }\end{array}$ & $\begin{array}{l}40 \% \\
40 \% \\
80 \%\end{array}$ \\
\hline $\begin{array}{l}\text { Fase IV (porcentaje de centros que la realizan) } \\
\text { Que herramienta utilizan para el cálculo de la } \\
\text { intensidad del ejercicio }\end{array}$ & $56 \%$ \\
\hline $\begin{array}{l}\text { * Borg } \\
\text { * Mets } \\
\text { * Edad } \\
\text { * } \mathrm{VO}_{2} \\
\text { * } \mathrm{FC}\end{array}$ & $\begin{array}{c}25 \% \\
100 \% \\
75 \% \\
0 \\
0\end{array}$ \\
\hline $\begin{array}{l}\text { Que profesionales realizan la fase IV } \\
\text { * Médico } \\
\text { * Enfermera } \\
\text { * Fisioterapeuta o kinesiólogo }\end{array}$ & $\begin{array}{l}75 \% \\
25 \% \\
75 \%\end{array}$ \\
\hline
\end{tabular}


Tabla 2. Recursos humanos en los diferentes centros de Chile

\begin{tabular}{|lr|}
\hline & $\%$ \\
\hline Cardiólogo & 100 \\
\hline Fisiatra o kinesiólogo & 85 \\
\hline Enfermeras entrenada & 85 \\
\hline Nutricionista & 85 \\
\hline Psicólogo & 42 \\
\hline Trabajadores sociales & 28 \\
\hline Otros* & 28 \\
\hline Médico general & 14 \\
\hline Médico deportólogo & 14 \\
\hline Psiquiatra & 0 \\
\hline Entrenamiento en reanimación cardiopulmonar & 100 \\
\hline
\end{tabular}

*kinesiólogo, técnico paramédico.

Tabla 3. Elementos de evaluación en las diferentes Fases de un programa de RC

\begin{tabular}{|lc|}
\hline & $\%$ \\
\hline Evaluación inicial & 100 \\
\hline Planificación de la terapia de ejercicio & 100 \\
\hline Consejos para la actividad física & 100 \\
\hline Entrenamiento para la toma de frecuencia & 100 \\
cardiaca & 100 \\
\hline Entrenamiento físico & 85 \\
\hline Apoyo psicológico & 42 \\
\hline Test de depresión & 14 \\
\hline Cuestionario de apnea del sueño & 100 \\
\hline Caminata de 6 minutos & 85 \\
\hline Prueba de esfuerzo convencional & 14 \\
\hline Prueba con VO & 14 \\
Otras pruebas de esfuerzo & 100 \\
\hline Educación nutricional & 100 \\
\hline Manejo de factores de riesgo & 42 \\
\hline Clínica de tabaquismo & 71 \\
\hline Determina el estado de tabaquismo & 100 \\
\hline Interviene en tabaquismo & 85 \\
\hline Atención a familiares & 56 \\
\hline Programa de salud cardiovascular de la mujer & 56 \\
\hline Seguimiento post al programa con consulta y \\
evaluación de FC de riesgo & 56 \\
\hline Realiza algún programa recreacional & 14 \\
\hline Yhai-Chi & 14 \\
Demostraciones de cocina & 14 \\
\hline
\end{tabular}

§Técnicas de relajación, caminatas, bailo terapia, Lian Gon. diaca. Ochenta y cinco porciento brindaba apoyo psicológico pero sólo $42 \%$ realizaba test de pesquisa de depresión. Sólo uno estaba actualmente aplicando cuestionario de apnea del sueño (14\%). Como evaluación inicial de la capacidad funcional y screening de isquemia miocárdica residual, la prueba preferida y realizada en $85 \%$ de los centros fue la ergométrica, encontrándose igualmente que sólo 2 centros estaban en condiciones de realizar test cardiopulmonar con consumo directo de oxígeno (14\%). El 100\% realizaba el test de caminata de 6 minutos. El 100\% intervenía y controlaba los factores de riesgo. El 71\% determinaba estado de tabaquismo, el 100\% lo abordaba con consejos y en $42 \%$ había derivación de pacientes fumadores a policlínicos de tabaco. Sólo 1 incluye a menores de 18 años (Tabla 3).

La mayoría (62,5\%) controlaba lípidos sanguíneos y glicemia. Sólo 14\% alguna vez solicitó PCR ultrasensible y lipoproteína a. Las tablas utilizadas como referencia para el manejo del colesterol elevado son la del ATP III ${ }^{19}$.

La composición corporal era determinada en $75 \%$ de los centros y los métodos empleados eran la medición de pliegues cutáneos $(83,3 \%)$ e impedanciometría (16,7\%). El 37,5\% pesquisaba y diagnosticaba síndrome metabólico, empleando los criterios del ATP III en $100 \%{ }^{19}$.

El ingreso a programas de RC y su cobertura era autofinanciada $75 \%$, siendo un sistema mixto (dependiente del hospital y en parte por la persona involucrada) en $25 \%$.

Si bien la derivación de los pacientes a estos programas es responsabilidad exclusiva de los médicos tratantes, los centros informaron que la principal barrera para tener una amplia concurrencia era la no derivación por parte de los mismos profesionales (56\%) y en un solo caso se hizo referencia a la falta de recursos económicos o falta de espacio en el centro. Ninguno opinó que ello se deba a la falta de recursos humanos. En relación al equipamiento, la mayoría mencionó contar con instrumental y base de datos electrónica, realizando investigación en sus servicios (75\%), con mecanismos de seguimiento en $75 \%$ de los centros (Tablas 3 y 5 ).

Los centros que poseen programas de Tabaco (25\%) eran manejados en $100 \%$ por médico. Cincuenta porciento contaba con programas de salud cardiovascular para la mujer.

Las actividades recreacionales paralelas se efec- 
Tabla 4. Tipos de patologías de los pacientes incluidos en los diferentes centros

\begin{tabular}{|lc|}
\hline Pacientes incluidos & \% \\
\hline Post Infarto del miocardio & 100 \\
\hline Post angioplastía & 100 \\
\hline Post puentes aorto-coronario & 100 \\
\hline Insuficiencia cardiaca & 85 \\
\hline Enfermedad valvular cardiaca & 85 \\
\hline Enfermedad arterial periférica & 85 \\
\hline Post trasplante cardiaco & 42 \\
\hline Sincope & 28 \\
\hline Otros* & 56 \\
\hline Menores de 18 años & 14 \\
\hline
\end{tabular}

*Otros: post implante marcapasos y cardiodesfibrilador, enfermedades pulmonares, pacientes con factores de riesgo para enfermedad cardiovascular.

túan en 50\%, thai-chi, yoga, caminatas, reuniones comunitarias. Sólo la mitad de los centros tiene programas de seguimiento posterior con consultas y control de los factores de riesgo, la que se realiza tanto con la concurrencia del paciente a la consulta médica o mediante llamadas telefónicas.

Todos los centros intervenían y rehabilitaban a pacientes tanto con cardiopatía isquémica como con otras patologías: insuficiencia cardiaca, valvulares, vasculares periféricos. Cuarenta porciento recibía pacientes con trasplante cardiaco y portadores de marcapasos, cardiodesfibrilador y sincopes neurocardiogénicos (Tabla 4).

Finalmente, la encuesta se interesó en averiguar sobre la calidad asistencial internándose en el tipo de servicios prestados en los centros involucrados.

Todos los encuestados mencionaron pertenecer a un servicio de cardiología, variando las prestaciones algunas cirugías cardiacas, ecocardiografia, electrofisiología, etc. (Tabla 6).

\section{Discusión}

Este estudio es el primer informe actual del estado de la RC en Chile. En Chile no se utiliza la medición rutinaria de apolipoproteínas ni score de calcio coronario. Los resultados obtenidos se basan en $89 \%$ de los centros existentes ${ }^{20-23}$.

La RC es considerada "gold standard" en el cuidado del paciente post-infarto de miocar$\operatorname{dio}^{24-28}$. No obstante, se demostró que son pocos
Tabla 5. Principales barreras de derivación

\begin{tabular}{|lc|}
\hline $\begin{array}{l}\text { Principal barrera detectada en la } \\
\text { atención de pacientes }\end{array}$ & $\%$ \\
\hline Falta de referencia al centro de RC & 56 \\
\hline Distancia del centro de rehabilitación & 28 \\
\hline Problemas músculo esqueléticos & 14 \\
\hline Falta de equipamiento & 0 \\
\hline Falta de recursos humanos & 0 \\
\hline Falta de espacio en el centro de rehabilitación & 0 \\
\hline Falta de recursos económicos & 0 \\
\hline
\end{tabular}

Tabla 6. Actividades y servicios prestados por el hospital o centro medico donde se encuentra el centro de rehabilitación cardiovascular

\begin{tabular}{|lr|}
\hline Cuantos centros tienen servicio de cardiología & \% \\
\hline Atención de infarto agudo del miocardio & 85 \\
\hline Centros con cirugía cardiovascular & 42 \\
\hline Centros con angiografía coronaria diagnóstica & 56 \\
\hline Centros con angiografía percutánea terapéutica & 56 \\
\hline Centros con electrofisiología invasiva & 71 \\
\hline Centros con ecocardiografía & 85 \\
\hline Centros con resonancia magnética cardiaca & 41 \\
\hline Centros con Tomografía para calcio coronario & 56 \\
\hline
\end{tabular}

los pacientes derivados a programas de $\mathrm{RC}$, sobre todo los que han padecido un evento coronario agudo (5\%).

No es fácil obtener datos exactos sobre la población que accede a servicios de RC luego del evento agudo ya que en nuestro país no es obligatoria su derivación y la RC no está cubierta por los seguros médicos.

Comparando nuestra serie con otros estudios publicados, vemos que Chile es uno de los países de Latinoamérica con menor porcentaje de derivación de pacientes a $\mathrm{RC}^{25}$.

Cotejados estos datos a nivel mundial, Suaya y col citan $20 \%$ de pacientes derivados a RC en Estados Unidos de Norteamérica $(22,1 \%$ en hombres vs $143 \%$ en mujeres ${ }^{29}$. En España, datos recientes hablan que no más de $4 \%$ de los pacientes reciben Rehabilitación Cardiaca tras un infarto 
(PREVEN SEC) $)^{30-35}$, mientras que en Austria, uno de los países en que la RC está más desarrollada, el 95\% de la población cardiaca tiene cobertura en Fase II, reduciéndose a $60 \%$ en Holanda y a $30 \%$ en Dinamarca ${ }^{36}$. En Chile los pacientes elegibles que acceden a RC son, en la mayoría, pacientes coronarios, habiendo homogeneidad en el número de sesiones planificadas y programas implantados, siendo realizadas mayormente en el sector privado. Un aspecto a resaltar es la programación de por lo menos 12 sesiones de RC. Esa duración mínima es la que ha demostrado ${ }^{37-39}$ un beneficio significativo: $47 \%$ menos de riesgo de muerte y $31 \%$ menos riesgo de infarto. Esta duración, concuerdan dichos autores, tiene gran influencia en la prevención de eventos cardiacos recurrentes en los 4-5 años posteriores a la Fase II de la RC. A pesar que los centros privados de nuestro país cuentan, en general, con la infraestructura adecuada, no se trabaja en la Fase I de la RC y el paciente comienza la Fase II algún tiempo después del evento cardiaco.

Se desconocen las cifras de todos aquellos pacientes a los que se les dio el alta y continúan realizando actividad física en clubes o por su cuenta, lo que es una de las grandes limitaciones de este trabajo. La educación y el control de los factores de riesgo cardiovascular es parte esencial de cualquier programa de $\mathrm{RC}^{40}$. En ese sentido, el estudio demostró que la mayoría de los centros de RC evalúa, estudia, estratifica y controla a sus pacientes y sus factores de riesgo, así como la aplicación de las pautas clásicas en el control de los mismos. Aunque la mayoría de los centros determina estado de tabaquismo, deberían ser más exigentes con el control, derivación y tratamiento del mismo. La apnea del sueño y la depresión son también considerados factores de riesgo cardiovascular $^{32,33}$. Sin embargo, sólo 1 está utilizando cuestionarios de apnea del sueño. En materia de análisis de laboratorio se pesquisa lo clásico (glicemia, perfil lipídico) pero aún no se ahonda en estudios como Apo A y Apo B, Lp a o PCRus.

Si bien la mayoría cuenta con base de datos y archivos, son muy escasas las publicaciones de esos datos. Más allá que ningún país contempla la situación óptima, este estudio sitúa a Chile en un lugar insuficiente y desigual frente a las naciones del primer mundo.

A la vista de los resultados no quedan dudas sobre la insuficiente implantación, desarrollo y potenciación de las unidades de RC existentes en Chile, más allá de los enormes beneficios que ella brinda en términos de costo-eficiencia ${ }^{41}$. Tampoco es desconocido que la rehabilitación cardiaca y prevención secundaria son maniobras terapéuticas altamente eficaces para el control de las enfermedades cardiovasculares y todas las guías internacionales la posicionan como Clase I dado sus mecanismos cardioprotectores mejorando la morbilidad y reduciendo la mortalidad ${ }^{42}$.

Es indispensable pensar y trabajar en conjunto desde la internación, la unidad de hemodinamia y cirugía cardiaca con las unidades de tratamiento al alta inmediata para evitar la pérdida de tiempo y deterioro de la calidad de vida del cardiópata. Luego de ello sería deseable mantener dichas medidas mejorando las prestaciones en las Fases III y IV, derivando a los pacientes a centros e instituciones debidamente preparados, bajo la supervisión de profesionales calificados y entrenados para mejorar el impacto socioeconómico que ellas conllevan y posicionar la RC de Chile en el sitial que ella debe ocupar.

Las estrategias deben venir dirigidas desde lo gubernamental a lo societario basándose en cinco pilares: desarrollo de los programas, difusión, implementación, puesta en marcha, mantenimiento y evaluación, tratando de concienciar a la población sobre la vital importancia de promover estilos de vida cardiosaludables ${ }^{40}$.

Agradecimientos: A los profesionales que gentil y desinteresadamente ocuparon su tiempo en responder el cuestionario y brindar los datos de sus centros: Klga. Claudia Román EU. Paula Soto, Klgo. Vicente Peña, Dr. Rafael Del Río, Dra. Inés Barquin, Dra. Mónica Acevedo, Dr. Rodrigo Ibáñez, Klgo. Mario Requena. Mención especial al departamento de prevención de la Sociedad Chilena de Cardiología.

\section{Referencias}

1. Dawber TR, Moore FE, Coronary heart disease in the Framingham Study. Am J Public Health 1957; 47: 4-24.

2. WHO. Cardiovascular Disease: prevention and control (site web). Obtenido de: http://www.who.int/cardiovascular_diseases/prevention_control/en/index

3. Yusuf S, Hawken S, Ounpuu S, Dans T, Avezum A, Lanas $\mathrm{F}$, et al. Effect of potentially modificable risk factors associated with myocardial infarction in 52 countries (The 
INTERHEART Study): case control study. Lancet 2004; 364: 937-52.

4. Comisión Honoraria para la Salud Cardiovascular. Informe de mortalidad y morbilidad por enfermedades cardiovasculares en el Uruguay 2003 (sitio web).

5. Curto S, Prats O, Ayestarán R. Investigación sobre factores de riesgo cardiovascular en Uruguay. Rev Méd Urug [online] 2004; (20), № 1 [citado el 23 de junio de 2009], p. 61-71.

6. Primera Encuesta Nacional de factores de Riesgo de Enfermedades Crónicas No Transmisibles. Ministerio de Salud Pública Uruguay. Dirección Nacional de Salud, junio 2007.

7. De Souza Ciorlia LA. Encuesta Internacional de Morbilidad por E cardiovasculares en un seguimiento de hasta 20 años. Colección “Trabajos Distinguidos”. Factores de Riesgo (SIIC). Volumen 6, Número 3, Abril 2008.

8. Dawber TR, Meadors GF, Moore FE. Epidemiological approaches to heart disease: the Framingham Study. Am J Public Health 1951; 41: 279-86.

9. Wilson PW, Castelli WP, Coronary risk prediction in adults (The Framingham Heart Study). Am J Cardiol 1987; 59: 691-4.

10. Encuesta nacional de salud 2010-2011, MINSAL, http:// www.wkd.cl/images/descargas/ENSalud_2010.pdf

11. O’ Donnell CJ, Elousa R. Factores de riesgo cardiovascular. Perspectivas derivadas del Framingham Heart Study. Rev Esp Cardiol 2008; 61: 299-310.

12- Rehabilitation of patients with cardiovascular diseases. Report of a WHO Expert Committee. Geneva, WHO, 1964 (WHO Technical Report Series, No 270).

13. Rehabilitation after cardiovascular diseases with special emphasis on developing countries. Report of a WHO Expert Committee. Geneva, WHO, 1993.

14. American Association of Cardiovascular and Pulmonary Rehabilitation. Guidelines of cardiac rehabilitation programs. $2^{\text {nd }}$. Ed. Champain, Illinois. Human Kinetics; 1999.

15. Burdiat G, Alonso P, Tejada J, Sandoya E, Vásquez H. Seguimiento clínico y paraclínico a 4 y 12 meses del alta en pacientes coronarios. Tema Libre XXV Congreso Sudamericano de Cardiología. Montevideo 25-28/09/10. Rev Urug Cardiol 2010; 25: 155.

16. Tomás Romero C. La Rehabilitación Cardiaca como punto de partida en la prevención secundaria de la enfermedad coronaria. Rev Med Chile 2000; 128: 923-34.

17. Borg G. Psychophysical bases of perceived exertion. Med Sports Exerc 1982; 14: 377-81.

18. Nazzal C, Campos P, Corbalán R, Lanas F, Bartolucci J, Sanhueza P, et al. Impacto del plan AUGE en el tratamiento de pacientes con infarto agudo $\mathrm{Al}$ miocardio con supradesnivel ST, en hospitales chilenos. Rev Med Chile [online] 2008; 136 (10): 1231-9.

19. National Cholesterol Education Program (NCEP). Expert Panel on Detection, Evaluation, and Treatment of High Blood Cholesterol in Adults. Adult Treatment Panel III (ATP III). Final report. Circulation 2002; 106 (25): 3143-421.

20. Korenfeld Y, Mendoza-Bastidas C, Saavedra L, MonteroGómez A, Pérez-Terzic C, Thomas R, et al. Current status of cardiac rehabilitation in Latin America and the Caribbean. Am Heart J 2009; 158: 480-7.

21. Illarraza Lomelí H, Herrera Franco R, Lomelí Rivas A, Zavala Ramírez J, Martínez Ramírez L, Ramos Becerri FJ, et al. Registro nacional sobre Programas de Rehabilitación Cardiaca en México (RENAPREC). Arch Cardiol Mex 2009; 79 (1): 63-72.

22. Márquez-Calderón S, Villegas Portero R, Briones Pérez de la Blanca E, Sarmiento González Nieto V, Reina Sánchez M, Saiz Hidalgo I, et al. Implantación y características de los programas de rehabilitación cardiaca en el Sistema Nacional de Salud español. Rev Esp Cardiol 2003; 56 (8): 775-82.

23. Vanhess L, McGee HM, Dugmore LD, A representative study of cardiac rehabilitation activities in European Union Members States: THE CARINEX SURVEY. (CARINEX Working Group: Cardiac Rehabilitation Information Exchange). J Cardiopulm Rehabil 2002; 22 (4): 264-72.

24. Suaya JA, Shepard DS, Normand ST, Use of cardiac rehabilitation by Medicare beneficiaries after myocardial infarction or coronary bypass surgery. Circulation 2007; 116: 1653-62.

25. Velazco JA, Cosin J, Maroto JM, Muñiz J, Casanovas JA, Plaza I, et al. Guías de práctica clínica de la Sociedad Española de Cardiología en prevención cardiovascular y rehabilitación cardiaca. Rev Esp Cardiol 2000; 53: 1095120.

26. Zwisler AD, Traeden UI, Videbaek J. Cardiac rehabilitation services in Denmark: still room for expansion. Scand J Public Health. 2005; 33: 376-83.

27. Suaya JA, Stason WB, Ades PA, Cardiac rehabilitation and survival in older coronary patients. J Am Coll Cardiol 2009; 5425-33.

28. Hammill BG, Curtis LH, Schulman KA, Whellan DJ. Relationship between cardiac rehabilitation and long-term risks of death and myocardial infarction among elderly. Medicare beneficiaries. Circulation 2010; 121: 63-70.

29. Lanas F, Avezum A, Bautista LE, Díaz R, Luna M, Islam $\mathrm{S}$, et al. Risk factors for acute myocardial infarction in Latin America: the Interheart Latin America study. Circulation 2007; 115: 1067-74. 
30. Lespérance F, Frasure-Smith N, Talajic M, Bourassa MG. Five-year risk of cardiac mortality in relation to initial severity and one-year changes in depression symptoms after myocardial infarction. Circulation 2002; 105: 104953.

31. Lett HS, Blumenthal JA, Babyak MA, Sherwood A, Strauman T, et al. Depression as a risk factor for coronary artery disease: evidence, mechanisms, and treatment. Psychosom Med 2004; 66: 305-15.

32. Parish JM, Somers VK. Obstructive sleep apnea and cardiovascular disease. Mayo Clin Proc 2004; 79: 1036-46.

33. Somers VK, White DP, Amin R, Abraham WT, Costa F, Culebras A, et al. Sleep apnea and cardiovascular disease: an AHA/ACC Foundation scientific statement from the American Heart Association Council for High Blood Pressure Research Professional Education Committee, Council on Clinical Cardiology, Stroke Council, and Council on Cardiovascular Nursing. Circulation 2008; 118: 1080-111.

34. Ades PA, Pashkow FJ, Nestor JR. Cost-effectiveness of cardiac rehabilitation alter myocardial infarction. J Cardiopulm Rehabil 1997;17: 222-31.

35. Briffa TG, Eckermann SD, Griffiths AD, Harris PJ, Rose M, Freedman SB, et al. Cost-effectiveness of rehabilitation alter an acute coronary event: a randomised controlled trial. Med J Aust 2005; 183: 450-5.

36. Giannuzzi P, Saner H, Björnstad H, Fioretti P, Mendes M, Cohen-Solal A, et al. Secondary Prevention Trough Cardiac Rehabilitation. Position paper of the Working Group on Cardiac Rehabilitation and Exercise Physio- logy of the European Society of Cardiology. Eur Heart J. 2003; 24: 1273-8.

37. Balady GJ, Williams MA, Ades PA, Bittner V, Comoss P, Foody JM, et al. Core Components of Cardiac Rehabilitation/Secondary Prevention Programs: 2007 Update. A Scientific Statement from the AHA and the AACVPR. Circulation 2007; 115: 2675-82.

38. Thomas RJ, King M, Lui K, Oldridge N, Piña IL, Spertus J. AACVPR/ACC/AHA 2007 Performances measures on cardiac rehabilitation for referral to and delivery of cardiac rehabilitation/secondary prevention services. Circulation 2007; 116: 1611-42.

39. Thomas RJ, King M, Lui K, Oldridge N, Piña IL, and AACVPR/ACC/AHA 2010 Update: Performances measures on cardiac rehabilitation for referral to cardiac rehabilitation/secondary prevention services. Circulation 2010; 122:1-7.

40. Piepoli MF, Corrà U, Benzer W, Bjarnason-Wehrens B, Dendale P, Gaita D, et al. Secondary prevention through cardiac rehabilitation: physical counselling and exercise training. Key components of the position paper from the Cardiac Rehabilitation Section of the European Association of Cardiovascular Prevention and Rehabilitation. Eur Heart J 2010; 31: 1967-76.

41. Franklin BA, Bronzheim K, Safety of medically supervised cardiac rehabilitation exercise therapy: a 16-year follow-up. Chest 1998; 114: 902-6.

42. Maroto Montero JM, Artigao Ramírez R, Morales Durán MD, Rehabilitación cardiaca en pacientes con infarto de miocardio. Rev Esp Cardiol 2005; 58 (10): 1181-7. 